\title{
Revisión de los métodos de medición del rendimiento académico en los estudiantes universitarios
}

\section{Review of methods of measuring academic achievement in university students}

\section{Juan C. Acosta-Jiménez ${ }^{1}$, Ildefonso Baldiris-Navarro ${ }^{2}$}

${ }^{1}$ M.Sc., Docente, Fundación Universitaria Tecnológico Comfenalco-Cartagena, Colombia. jacosta@tecnologicocomfenalco.edu.co

${ }^{2}$ M.Sc., Docente, Servicio Nacional de Aprendizaje-SENA. Cartagena, Colombia. ibaldirisn@sena.edu.co

Recibido: $15 / \mathrm{feb} / 2020$ Revisado: 30/abr/2020

Aceptado: 30/may/2020 Publicado: 30/jul/2020

Resumen Las Instituciones de Educación Superior, son empresas que brindan servicios educativos, ofreciendo programas académicos a nivel tecnológico, universitario, de formación continua y posgrados, diseñados para atender necesidades sentidas del sector social y productivo, mediante un proceso integral. En el desarrollo del presente escrito, se realizó una revisión descriptiva sobre los distintos métodos estadísticos usados para determinar las causas que han generado el problema de deserción escolar y el bajo rendimiento académico de los estudiantes, lo cual proporciona al lector una puesta al día sobre conceptos y herramientas de gran interés, para establecer acciones de precaución y mejora continua en los procesos académicos de enseñanza aprendizaje.

Palabras claves rendimiento académico; deserción estudiantil; métodos estadísticos.

Abstract The Higher Education Institutions are companies that provide educational services, offering academic programs at the technological, university, continuing education and postgraduate levels, designed to meet the needs of the social and productive sector through a comprehensive process. In the development of this paper, a descriptive review was made of the various statistical methods used to determine the causes that have generated the problem of dropout and the low academic performance of students, which provides the reader with a day on concepts and tools of great interest to establish actions of precaution and continuous improvement in the academic processes of teaching learning.

Keywords academic performance; student dropout; statistical methods.

Teknos Revista Científica. | Volumen 20 No.1 - Julio 2020 | ISSN 1900-7388 (papel) | ISSN 2539-2190 (digital)

DOI: https://doi.org/10.25044/issn.2539-2190 


\section{Introducción}

Actualmente, una de las preocupaciones que viven las Universidades Colombianas, junto con los sistemas educativos de la región y del mundo, es el bajo rendimiento académico de sus estudiantes (Guzmán, Muriel, \& Franco, 2009), lo cual se ve reflejado constantemente en los prolongados tiempos para graduarse, la reprobación de semestres o la no búsqueda de la excelencia en los estudios, los cuales son indicadores de calidad que representan el cumplimiento de objetivos establecidos, ya sea por la institución o en el programa que se esté cursando.

Muchos autores consideran que el rendimiento académico establece el nivel de aprendizaje alcanzado por un educando, el cual define la eficacia en la obtención de los objetivos curriculares establecidos en cada asignatura.

Páez y Castrillón (2015), Sánchez, Carvajal y Saggiomo (2016) y Valencia, Pareja y Montoya (2014) argumentaron que existen muchos factores que pueden afectar este proceso, puesto que puede implicar una condición de fracaso en el estudiante, lo que en muchos casos disminuiría las aspiraciones propias 0 institucionales, implicando un receso académico o a las últimas la deserción del educando.

En la mayoría de las Universidades Colombianas se implementa mediante un guarismo basado en el sistema de base cinco; es decir, las notas varían de 0 a 5 puntos, donde el puntaje de 3 o más es aprobatorio; por lo cual existe la necesidad de indagar los factores que impactan el rendimiento académico de los estudiantes de parte de las Instituciones de Educación Superior, sus políticas establecidas y los respectivos resultados obtenidos.

Dentro del contexto educativo es frecuente escuchar sobre el fracaso estudiantil, por la insatisfacción con los contenidos impartidos y reprobación de semestres, entre otros; la necesidad de predecir cuál será el rendimiento académico y la relación que guarda éste con factores internos o externos de cada universidad, qué herramientas y métodos se han utilizado y cual ha tenido mejores resultados, origina la presente revisión de literatura, en donde se mostraran los principales métodos estadísticos utilizados en el país, junto con sus respectivos resultados.

\section{Método}

Este artículo de revisión, es un estudio bibliográfico en el que se recopila, analiza y sintetiza la información publicada sobre cierto tema. El método de revisión es descriptivo (Vera, 2009), porque proporciona al lector una puesta al día sobre las herramientas estadísticas utilizadas en la medición del desempeño académico en los distintos tipos de instituciones educativas.

En este caso, para elaborar dicha revisión, se tomaron como referencia artículos consultados en revistas de $S$ copus ${ }^{\circledR}$, la cual es una base de datos de referencias bibliográficas y citas de la empresa Elsevier ${ }^{\circledR}$. La estrategia de búsqueda fue colocar descriptores y palabras claves tales como: rendimiento académico, deserción estudiantil y métodos estadísticos. La organización de la información se llevó a cabo mediante el gestor de referencias bibliográficas Mendeley®. Finalmente se procedió con la redacción del presente documento.

\section{Revisión de la literatura}

A nivel internacional, se ha estudiado la transición de un joven de secundaria a la Universidad, como el caso de una investigación realizada en España (González et al., 2011), en donde se caracterizaron los factores personales de sus alumnos, específicamente se indagó por variables como la edad, si trabajaban o no, si han tenido problemas familiares. En otros casos, se ha indagado sobre la incidencia de los factores psicológicos, en donde se ha concluido que los estudiantes con creencias de autoeficacia más altas, asientan más intención de alcanzar sus metas (García \& Segundo, 2001).

También se tiene información de que el modelo educativo de la institución, impacta en los resultados que obtiene un estudiante (Solís et al., 2015), hasta la 
calidad de un buen desayuno repercutiría en dicho proceso (Fernández et al., 2008). Por otro lado, no todos los estudios son comparables debido a que evalúan diferentes variables, como sucedió en la Universidad Nacional de Salta en Argentina, en donde se observó que el rendimiento académico no tenía relación con la actividad laboral, el tipo de escuela y la procedencia (Sanguinetti et al., 2013).

En la Universidad Nacional de la Plata en Argentina, se encontró que los estudiantes que trabajan en áreas relacionadas con su programa de estudio obtienen mejores resultados académicos. (Carella, 2009).

En el ámbito nacional, varias universidades han mostrado interés sobre el desempeño académico de sus estudiantes, por ejemplo: el programa de ingeniería de sistemas de una institución universitaria de Manizales, analizó mediante métodos estadísticos descriptivos, las estrategias de aprendizaje utilizadas por los estudiantes con bajo rendimiento académico de primer y segundo semestre (Cortés et al., 2006). En la Universidad Libre de Barranquilla, se evidenció que para evaluar el rendimiento estudiantil es necesario indagar por un gran número de variables, no solo las relacionadas con las notas obtenidas, sino sus antecedentes académicos previos. (Mendoza, 2013).

La importancia de conocer las causas del bajo rendimiento académico de un estudiante no es exclusiva de las instituciones de educación superior, pues a nivel de primaria se han identificado que los principales problemas asociados al rendimiento escolar de los niños son relacionados con hiperactividad, desatención, tristeza, preocupación y conductas disruptivas en los salones de clases (Shapiro, 2011). En la secundaria, la preocupación también es inminente especialmente en el área de las matemáticas (Vitola, 2015), aunque algunos autores afirman que una de las causas es la desigualdad digital que vivimos en los países subdesarrollados (Castaño, 2010).

\section{Métodos estadísticos usados para calcular el rendimiento académico}

\subsection{Métodos descriptivos}

La principal y más usada manera de presentar resultados sobre estudios investigativos a nivel general, es la estadística descriptiva, la cual conlleva a la utilización de medidas para caracterizar una variable de interés, entre las más comunes encontramos: medidas de tendencia central como medias, medianas y moda (Torres \& Rodríguez, 2006) (Antonio \& Murcia, 2015), medidas de variabilidad como varianzas, desviaciones estándar, coeficientes de variación (Soares, Antunes, \& Aguiar, 2015). También se hacen referencias del uso de tablas de contingencias (Valencia et al., 2014), junto con medidas de colocación o posición como cuartiles y percentiles (Martín, 2007), finalmente las principales representaciones gráficas: diagramas circulares, barras, histogramas y pictogramas que siempre se tienen en cuenta, por su facilidad de interpretación (García, Muñoz, \& Arras, 2009) (Cendales, Vargas, \& Barbosa, 2013).

\subsection{Métodos inferenciales}

La implementación de estas técnicas en términos generales, buscan establecer pronósticos para una variable de interés de una población con base a una muestra representativa de la misma (Walpole, Myers, \& Myers, 2012). Dentro de las principales herramientas más usadas en estudios de investigación educativos, están las pruebas de hipótesis y la estimación de parámetros por intervalos de confianza.

$\mathrm{Se}$ han realizado estudios de pruebas de significancia en donde se argumenta que el rendimiento de un estudiante depende si la institución de donde proviene es pública o privada (Solís et al., 2015), también la ansiedad conductual intervendría en sus notas (Hernández-Pozo. et al., 2008). Otras pruebas han mostrado que la ayuda de estudiantes tutores compañeros de clases de estudiantes con bajo rendimiento, aumenta las posibilidades de mejora (Martin, Tirado, Ortega, \& Díaz, 2011), caso contrario ocurre por la depresión debida a problemas personales (Campo \& Rueda, 2005). 
En los programas de ciencias humanas como psicología, trabajo social y derecho, se ha aplicado mucho la regresión lineal en busca de correlacionar variables que inciden de una $u$ otra manera al desempeño estudiantil, para esto se aplican y analizan test de conducta (Valle \& Urquijo, 2015), test de personalidad (Rodríguez, Acosta, \& Torres, 2013), evaluaciones diagnósticas (Muñóz, Leenen, \& Fortoul, 2014), conocimientos previos (Elvira \& Pujol, 2012), trastornos de ansiedad (Caballero, Abello, \& Palacio, 2007), las relaciones familiares (Caso \& Hernández, 2007), conceptos sobre las inteligencias múltiples (Velásquez, Puentes, \& Castro, 2014), manejo del estrés académico (Pozos, Preciado, Acosta, Aguilera, \& Delgado, 2014), nivel metacognitivo del estudiante (Landines, Escorcia, Moreno, \& Palacio, 2015), voluntad para estudiar (Broc, 2011), y las competencias emocionales (Leticia, González, \& García, 2013).

\subsection{Métodos multivariados}

El uso del análisis estadístico multivariado es una estrategia muy usada para modelar el estado académico de los alumnos, como es el caso de la Universidad de la República en Uruguay, que desarrolló una investigación correlacional considerando como factores explicativos: las variables sociodemográficas, el rendimiento previo de sus estudiantes y la percepción de su propia capacidad (Ayán \& Díaz, 2011).

Otra de las metodologías que se han usado, es el Análisis envolvente de datos, definida como una técnica de investigación de operaciones, específicamente de programación lineal. Para el caso educativo, se propone aplicar esta técnica para calcular el rendimiento relativo de un estudiante respecto al grupo al cual se encuentra matriculado. (Mendoza, 2013).

La implementación del Análisis Factorial Exploratorio, ha tenido el objetivo de explorar la presencia de variables latentes que inciden el rendimiento académico, mientras que el Análisis Factorial Confirmatorio, ha sido utilizado para relacionar la variable: logro académico, con distintos patrones de pensamiento (Gaeta, Cavazos, Sánchez, Rosário, \& Hgemann, 2015). Otras variables estudiadas con este método son la autorregulación, aptitudes intelectuales generales, motivación y creatividad (Ordaz \& Reyes, 2014), los factores cognitivos y comportamentales (Marín, Zueck, \& Cuadras, 2011), tiempo de estudio y enfoques de aprendizaje (Sánchez, 2007).

Planck (2014), afirma que existe una fuerte relación entre el desempeño académico y los factores personales y sociales. En el primero de ellos se hace alusión a la inteligencia, las aptitudes, la asistencia a clases, el género, la nota de acceso a la universidad. Mientras que en el segundo se tienen en cuenta el entorno familiar, el contexto socioeconómico, las variables demográficas tales como: sexo, edad, estado civil y la escolaridad de los padres. Luego, se establece un modelo de regresión lineal múltiple, el cual también ha sido utilizado para predecir resultados en las universidades (Pereira, Hernández, \& Gómez, 2011), y en colegios (Reyes et al., 2014).

Otro tipo de regresión es la logística en donde se consideran las variables predictoras como atributos y establece la relación con el factor de interés (Lapresa, Arana, Anguera, Pérez, \& Amatria, 2016), varios autores al usar esta herramienta estadística, establecen que el hábito de estudio, el entorno del estudiante, la elección del programa, la confianza en la institución, la actitud hacia las asignaturas, repercuten en que gane o pierda las materias cursadas (Díaz \& Giraldo, 2011). En otra pesquisa realizada en un programa de Psicología, se concluyó que el rendimiento académico dentro de la carrera se contempla con un modelo logístico, al analizar variables de capacidad intelectual, psicopatología, alexitimia y sociodemográficas (Moral, 2006).

El análisis discriminante se realiza con fines descriptivos y se enfoca en determinar cuáles variables son las que más diferencian a los grupos y cuáles son importantes (Pérez \& Támara, 2012). Uno de estos estudios concluyó que el estudiante universitario de éxito es un estudiante que utiliza estrategias motivacionales de tipo intrínseco, que autorregula su estudio planificando y revisando el 
proceso, y que utiliza estrategias de elaboración de anclaje que facilitan el aprendizaje significativo (Martín, García, Torbay, \& Rodríguez, 2008).

En los estudios del rendimiento académico universitario, también se ha utilizado la Característica Operativa del Receptor o curvas ROC, la cual se implementó dentro de la teoría de la decisión y su principal aplicación se da en la resolución de problemas de detección de señales. En el ámbito estudiantil, se considera un grupo de alumnos y a partir de una variable continua tal como nota final de la asignatura y se discriminan a cada uno de dichos estudiantes, en una de las dos categorías de éxito o fracaso (María, Molina, \& Sánchez, 2004).

\section{Conclusiones}

En Colombia en todas las instituciones en donde se imparte enseñanza para la educación primaria, secundaria, técnica, tecnológica y profesional, se están presentando altos índices de deserción académica que, junto con los bajos puntajes de los estudiantes en pruebas estandarizadas 0 evaluaciones, han creado preocupación dentro de las directivas docentes y por ende conlleva a la toma de medidas que subsanen dichos problemas. Para lograrlo, se han establecido estudios de análisis de resultados académicos, en busca de identificar las causas que generan el efecto negativo de que un estudiante abandone los estudios 0 que sus calificaciones mejoren.

Para el tratamiento de los datos recolectados por medio de aplicativos o test, las herramientas estadísticas juegan un papel fundamental, las más usadas son las descriptivas, inferenciales y el análisis multivariado. Sin embargo, ellas solo sintetizan la data recopilada pero las acciones correctivas y de mejora las debe realizar la institución de acuerdo con sus necesidades y la capacidad de operación.

En otros países en condiciones como el nuestro, los llamados subdesarrollados, también se presentan las mismas dificultades y se evidencia el esfuerzo por mejorar la calidad académica. Dentro de las causas comunes identificadas para que un estudiante universitario deserte, están la perdida de asignaturas de ciencias básicas, problemas financieros de manutención y pago de derechos pecuniarios, afectaciones emocionales y familiares.

\section{Referencias}

Antonio, J., \& Murcia, M. (2015). Modelo predictivo para la mejora de la percepción de competencia y rendimiento académico en estudiantes universitarios Predictive model to improve the competence perception and academic performance in colleges, 13(2), 173-188.

Ayán, M. N. R., \& Díaz, M. Á. R. (2011). Indicadores de rendimiento de estudiantes universitarios: Calificaciones versus créditos acumulados. Revista de Educacion, 355, 467-492. https://doi.org/104438/1988-592X-RE-2011-355-033

Broc, M. (2011). Voluntad para estudiar, regulación del esfuerzo, gestión eficaz del tiempo y rendimiento académico en alumnos universitarios, 29, 171-185.

Caballero, C. C., Abello, R., \& Palacio, J. (2007). Relación del burnout y el rendimiento académico con la satisfacción frente a los estudios en estudiantes universitarios. Avances En Psicología Latinoamericana, 25(2), 98-111.

Campo, A., \& Rueda, G. (2005). Percepción de rendimiento académico y síntomas depresivos en estudiantes de media vocacional de Bucaramanga, Colombia. Archivos de Pediatría ..., 76(1), 21-26. Retrieved from http://www.scielo.edu.uy/scielo.php?pid=S000405842005000100005\&script=sci_arttext\&tlng=pt

Carella, L. (2009). Educación universitaria: medición del rendimiento académico a través de fronteras de eficiencia, 40.

Caso, J., \& Hernández, L. (2007). Variables que inciden en el rendimiento académico de adolescentes mexicanos. Revista Latinoamericana de Psicologia, 39(3), 487-501.

Castaño, J. (2010). La desigualdad digital entre los alumnos universitarios de los países desarrollados y su relación con el rendimiento académico, 7.

Cendales, B., Vargas, E., \& Barbosa, C. (2013). Factores psicológicos asociados al desempeño académico en los cursos universitarios de estadística: diferencias por sexo y área de titulación. (Spanish). Avances En Psicología Latinoamericana, 31(2), 363-375. Retrieved from http://search.ebscohost.com/login.aspx?direct=true\&d $b=f u a \& A N=90510267$ \&lang=es\&site=ehost-live

Cortés, C., García, G., \& García, I. (2006). Estrategias de 
aprendizaje utilizadas por los estudiantes con bajo rendimiento académico de 10 y 20 semestre de ingeniería de sistemas y telecomunicaciones de la universidad de manizales, 26-36.

Díaz, H., \& Giraldo, J. (2011). Un modelo conceptual para el análisis del, 115-125.

Elvira, M., \& Pujol, L. (2012). Autorregulación y rendimiento académico en la transición secundaria-universidad. Revista Latinoamericana de Ciencias Sociales, Niñez Y Juventud, 10(1), 367-378. Retrieved from http://revistaumanizales.cinde.org.co/index.php/Revist a-Latinoamericana/article/viewArticle/612

Fernández, M. I., Aguilar, V. M., Vega, M., Martínez, P. C., \& Victorina, A. M. (2008). Relación entre la calidad del desayuno $y$ el rendimiento académico en adolescentes de Guadalajara (Castilla-La Mancha). Nutr Hosp, 23(4), 383-387.

Gaeta, M. L., Cavazos, J., Sánchez, A. P., Rosário, P., \& Hgemann, J. (2015). Propiedades psicométricas de la versión mexicana del cuestionario para la evaluación de metas académicas (CEMA). Revista Latinoamericana de Psicologia, 47(1), 16-24. https://doi.org/10.1016/S0120-0534(15)30002-9

Garcia, A., Muñoz, V., \& Arras, M. (2009). Competencias en tic y rendimiento académico en la universidad: Diferencias por género, 10.

García, M., \& Segundo, M. S. (2001). El rendimiento académico en el primer curso universitario. X Jornadas de Economía de La ..., 435-446. Retrieved from $\mathrm{ftp}: / / \mathrm{ftp}$.puce.edu.ec/Facultades/CienciasEducacion/M aestria/CienciasEducacion/Economía de la Educación/Garcia_SanSegundo.pdf

González, M. Á., Gazo, P. F., \& Fonseca, M. T. (2011). La problemática de la transición bachillerato-, 22, 15-27.

Guzmán, R. C., Muriel, D. D., \& Franco, G. J. (2009). Deserción estudiantil en la educación superior colombiana. Metodología de seguimiento, diagnóstico y elementos para su prevención. Retrieved from http://www.mineducacion.gov.co/sistemasdeinformaci on/1735/articles-254702_libro_desercion.pdf

Hernández-Pozo., M. del R., Coronado, O., Araujo, V., \& Cerezo, S. (2008). Desempeño académico de universitarios en relación con ansiedad escolar y autoevaluación. Acta Colombiana de Psicología, 11, 1323. https://www.redalyc. org/articulo.oa?id=79811102

Landines, K. C., Escorcia, D., Moreno, M., \& Palacio, J. (2015). Metacognición, escritura y rendimiento académico en universitarios de Colombia y Francia. Avances En Psicología Latinoamericana, 34, 1-42.

Lapresa, D., Arana, J., Anguera, M. T., Perez, J. I., \&
Amatria, M. (2016). Application of logistic regression models in observational methodology: game formats in grassroots football in initiation into football. Anales De Psicologia, 32(1), 288-294. https://doi.org/10.6018/analesps.31.3.186951

Leticia, M., González, G., \& García, C. L. (2013). Competencias emocionales y rendimiento académico en Emotional competencies and academic achievement in university students.

María, J., Molina, V., \& Sánchez, M. (2004). Estudio del rendimiento académico universitario basado en curvas Roc, 22, 327-340.

Marín, M. M., Zueck, C., \& Cuadras, G. G. (2011). Conductas académicas en universitarios de primer first grade university student' s psychometric analysis of an self.

Martin, F., Tirado, J., Ortega, S., \& Díaz, V. (2011). Prevención del fracaso académico universitario mediante tutoría entre iguales. Revista Latinoamericana de Psicologia, 43(1), 59-71.

Martín, I. (2007). Estrés académico en estudiantes universitarios. Apuntes de Psicología, 25(Inhelder 1974), 87-100.

Martín, García, L., Torbay, A., \& Rodriguez, T. (2008). Estrategias de aprendizaje y rendimiento académico en estudiantes universitarios. International Journal of Psychology and Psychological Therapy, 8(3), 401412.

Mendoza, A. (2011). Propuesta para la medición del rendimiento académico de los estudiantes de la universidad libre utilizando análisis envolvente de datos (dea), (9), 7-10.

Mendoza, A. (2013). Propuesta para la medición del rendimiento académico de los estudiantes de las universidades utilizando analisis envolvente de datos.

Moral, J. (2006). Predicción del rendimiento académico universitario. Perfiles Educativos, 28, 38-63.

Muñoz, A., Leenen, I., \& Fortoul, T. I. (2014). Correlación entre la evaluación diagnóstica y el rendimiento académico de los estudiantes de medicina. Investigación En Educación Médica, 3(10), 85-91. https://doi.org/10.1016/S2007-5057(14)72731-0

Ordaz, villegas G., \& Reyes, L. I. (2014). Development of an academic self concept for adolescents ( asca) scale académico para adolescentes ( aapa ). Journal of Behavior, Health \& Social Issues, 5(2), 117-130. https://doi.org/10.5460/jbhsi.v5.2.42304

Páez, M., \& Castrillón, J. (2015). Inteligencia emocional y rendimiento académico en estudiantes universitarios undergraduate students, 32.

Pereira, C., Hernández, G., \& Gómez, I. (2011). frente al

Teknos Revista Científica. | Volumen 20 No.1 - Julio 2020 | ISSN 1900-7388 (papel) | ISSN 2539-2190 (digital)

DOI: https://doi.org/10.25044/issn.2539-2190 
rendimiento académico universitario $O$ valor preditivo dos testes de Estado Palavras-chave, 14(1), 51-65.

Pérez, F., \& Támara, A. (2012). Análisis Discriminante Como Seleccionador De Variables, 20(27), 103-118.

Planck, B. (2014). Factores determinantes del rendimiento académico de los estudiantes de la Universidad de Atacama, XL, 25-39.

Pozos, E., Preciado, M., Acosta, F. M., Aguilera, M., \& Delgado, D. (2014). Psicología Educativa. Psicología Educativa, 20(1), 47-52. https://doi.org/10.1016/j.pse.2014.05.006

Reyes, R., Flaviano, C., Jaimes, G., Julián, F., Hernández, A., \& Sánchez, F. (2014). Un modelo empírico para explicar el desempeño académico de estudiantes de bachillerato. Revue d'Epidémiologie et de Santé Publique, 36(146), 45-62. https://doi.org/10.1016/S0185-2698(14)70127-8

Rodríguez, J., Acosta, M., \& Torres, N. (2013). Personalidad, aprendizaje y rendimiento académico en medicina. Investigación En Educación Médica, 2(8), 193-201. https://doi.org/10.1016/S2007-5057(13)72712-1

Sanchez, E. (2007). Contexto socioeconómico, percepción del contexto educativo y tiempo de estudio en relación con losy tiempo de estudio en relación con los resultados de aprendizaje en la educación superior, 17(30), 31-46.

Sánchez, F., Carvajal, F., \& Saggiomo, C. (2016). Self-talk and academic performance in undergraduate students, 32, 139-147.

Sanguinetti, J., López, R., Vieta, M., Berruezo, S., \& Chagra, C. (2013). Factores relacionados con el rendimiento académico en alumnos de fisiopatología. Investigación En Educación Médica, 2(8), 177-182. https://doi.org/10.1016/S2007-5057(13)72710-8

Shapiro, B. K. (2011). Bajo rendimiento escolar: una perspectiva desde el desarrollo del sistema nervioso. Revista Médica de Clínica Las Condes, 22(2), 218225. https://doi.org/10.1016/S0716-8640(11)70416-3

Soares, J. A. P., Antunes, H. R. L., \& Aguiar, C. F. dos S. (2015). Prática desportiva e sucesso escolar de moças e rapazes no ensino secundário. Revista Brasileira de
Ciências Do Esporte, 37(1), 20-28. https://doi.org/10.1016/j.rbce.2013.06.002

Solís, L., Baeza, J., Mena, D., \& Rodríguez, M. (2015). Estudio Comparativo de dos Modelos Educativos basado en los Resultados del Rendimiento Académico de los Alumnos de Licenciatura en Ingeniería. Formación Universitaria, 8(3), 47-56. https://doi.org/10.4067/S0718-50062015000300006

Torres, V. L. E., \& Rodriguez, S. N. Y. (2006). Academic performance and the family context in college students. Enseñanza E Investigación En Psicología, 11, 255-270. Retrieved from http://www.orienta.org.mx/docencia/Docs/Sesion-78/Torres.pdf

Valencia, A. M., Pareja, Á. M., \& Montoya, D. A. (2014). Trastornos neuropsiquiátricos en estudiantes universitarios con bajo rendimiento académico de una universidad privada de Medellín. Neuropsychiatric Disorders in a Sample of University Students with Academic Lower Performance of a Private Medellin University., 7(1), 69-78. Retrieved from http://search.ebscohost.com/login.aspx?direct=true\&d $b=$ fua\&AN $=98679932$ \&lang=es\&site $=$ ehost-live

Valle, M. V. Del, \& Urquijo, S. (2015). Relaciones de las estrategias de codificación mnésica y la capacidad de aprendizaje con el desempeño académico de estudiantes universitarios. Psicología Educativa, 21(1), 27-37. https://doi.org/10.1016/j.pse.2015.02.004

Velásquez, T., Puentes, A., \& Castro, H. (2014). Inteligencias múltiples y su relación con el rendimiento académico en Ingeniería de sistemas. Educación en Ingeniería,Vol 9, 44-52.

Vera, C. O. (2009). Cómo escribir artículos de revisión. Revista Médica La Paz, 15(1), 63-69.

Vitola, L. (2015). Regresión logística: una aplicación en variables que inciden en rendimiento académico. Revista Educación y Desarrollo Social, 118-132.

Walpole, R. E., Myers, R. H., \& Myers, S. L. (2012). Probabilidad y Estadística para Ingeniería y Ciencias (Novena). México: Pearson. 